\title{
Rolls of the internal gravity waves in the Earth's atmosphere
}

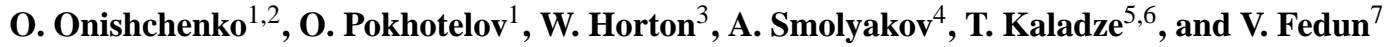 \\ ${ }^{1}$ Institute of Physics of the Earth, 10 B. Gruzinskaya, 123995 Moscow, Russian Federation \\ ${ }^{2}$ Space Research Institute, 84/32, Profsoyuznaya str., 117997 Moscow, Russian Federation \\ ${ }^{3}$ Institute for Fusion Studies, University of Texas at Austin, Austin, Texas, USA \\ ${ }^{4}$ University of Saskatchewan, Saskatchewan, S7N 5E2, Canada \\ ${ }^{5}$ I. Vekua Institute of Applied Mathematics, Tbilisi State University, Tbilisi, Georgia \\ ${ }^{6}$ Physics Department, Government College University, Lahore 54000, Pakistan \\ ${ }^{7}$ Space Systems Laboratory, Department of Automatic Control and Systems Engineering, \\ University of Sheffield, Sheffield, S1 3JD, UK
}

Correspondence to: V. Fedun (v.fedun@ sheffield.ac.uk)

Received: 28 October 2013 - Revised: 19 December 2013 - Accepted: 28 January 2014 - Published: 25 February 2014

\begin{abstract}
The effect of the wind shear on the roll structures of nonlinear internal gravity waves (IGWs) in the Earth's atmosphere with the finite vertical temperature gradients is investigated. A closed system of equations is derived for the nonlinear dynamics of the IGWs in the presence of temperature gradients and sheared wind. The solution in the form of rolls has been obtained. The new condition for the existence of such structures was found by taking into account the roll spatial scale, the horizontal speed and wind shear parameters. We have shown that the roll structures can exist in a dynamically unstable atmosphere.
\end{abstract}

Keywords. Electromagnetics (wave propagation)

\section{Introduction}

The energy transfer from the Earth's lithosphere to the atmosphere and ionosphere is a fundamental problem of geophysics and applied research. Internal gravity waves (IGWs) play an important role in such a process. For example, IGWs, propagating upward from the Earth's surface to the upper atmosphere and ionosphere, are able to carry a large amount of energy and momentum. These waves are crucial in atmosphere convection, generation of atmospheric turbulence and may affect global circulation. IGWs and related nonlinear structures are widely observed in the upper and lower layers of the atmosphere as well as in the lower ionosphere, (see Fritts and Alexander, 2003; Swenson and Espy, 1995; Picard et al., 1998; Lu et al., 2009; Mitchell and Howells, 1998; Šindelářová et al., 2009, for details).
The properties of IGWs strongly depend on the vertical temperature gradient. IGWs and atmosphere are convectively unstable when the square of the Brunt-Väisälä (buoyancy) frequency

$\omega_{g}^{2}=g\left(\frac{\gamma-1}{\gamma H}+\frac{1}{T_{0}} \frac{\mathrm{d} T_{0}}{\mathrm{~d} z}\right)$

is negative. Here $g$ is the gravitational acceleration, $\gamma$ the ratio of specific heats, $H$ the reduced height of the atmosphere, and $T_{0}$ the temperature. From Eq. (1), it follows that atmosphere layers with negative super-adiabatic temperature gradient, i.e.,

$\left|\frac{1}{T_{0}} \frac{\mathrm{d} T_{0}}{\mathrm{~d} z}\right|>\frac{\gamma-1}{\gamma H}$

are convectively unstable. In the presence of sheared winds, the development of the dynamic (Kelvin-Helmholtz) instability generally occurs when the Richardson number

$R i=\frac{\omega_{g}^{2}}{U^{\prime 2}}$

is smaller than some critical value, which depends on the velocity profile of the wind. Here $U$ is the wind velocity and $U^{\prime}=\mathrm{d} U / \mathrm{d} z$ is the wind shear. The necessary condition for the dynamic instability of steady, plane-parallel flow is $R i<0.25$ (Dutton, 1986). The atmosphere parameters, and instability excitation, depend on season and altitude. Observations of the mesopause region (i.e., $80-105 \mathrm{~km}$ ) show that the probability of convective and dynamic instability ranges 
from 1 to 2 and from 3 to 10 percent, respectively (Sherman and Che, 2006; Zhao, 2003). The amplitude of IGWs propagating upward throughout the various regions of the Earth's atmosphere and ionosphere grow with height and become unstable due to parametric instability as a result of resonant wave-wave interaction. Such parametric generation of largescale zonal structures by high-frequency finite-amplitude IGWs has been investigated recently by Horton et al. (2008) and Onishchenko and Pokhotelov (2012). The existence of the nonlinear IGWs in the form of two-dimensional smallscale vortices (i.e., roll-type structures) has been reported by Kaladze and Tsamalashvili (1997) and Stenflo and Shukla (2009). Recently, Onishchenko et al. (2013) have shown that the finite temperature gradient can substantially modify the condition for the existence of vortex-type structures obtained in previous works.

Of particular interest in the studies of IGWs is to investigate the influence of the wind shear on the nonlinear dynamics of high-frequency IGWs, which have the intrinsic frequency $\omega$ in the range $\left|\omega_{g}\right|>|\omega| \gg|f|$. Here, $f=2 \Omega \sin \phi$ is the Coriolis parameter, $\Omega$ the Earth rotation rate, and $\phi$ the latitude. In the present paper, we investigate the nonlinear structures associated with these waves.

The paper is organized as follows. In Sect. 2, we derive the reduced set of nonlinear hydrodynamic equations that describe the behavior of IGWs and illustrate the effect of shear wind in such an atmosphere. In Sect. 3, we investigate the solution of these equations in the form of convective cells. Our conclusions are found in Sect. 4.

\section{Reduced hydrodynamic equations}

The nonlinear dynamics of high-frequency IGWs will be studied in the local Cartesian coordinate system $(x, y, z)$, where the $z$ axis coincides with the vertical direction. The equation of momentum conservation and the transport equation for potential temperature read as follows:

$$
\begin{aligned}
\rho \frac{\mathrm{d} \boldsymbol{u}}{\mathrm{d} t} & =-\nabla p+\rho \boldsymbol{g}, \\
\frac{\mathrm{d} \Theta}{\mathrm{d} t} & =0
\end{aligned}
$$

where

$$
\Theta=p^{1 / \gamma} / \rho
$$

is the potential temperature, $\rho$ the density, $p$ the atmosphere pressure, $\boldsymbol{u}$ the fluid velocity, $\mathrm{d} / \mathrm{d} t=\partial / \partial t+\boldsymbol{u} \cdot \boldsymbol{\nabla}$ the advective (Euler) time derivative, $\boldsymbol{g}=-g \hat{z}$ the gravitational acceleration vector, and $\hat{z}$ the unit vector along the $z$ axis. The system of Eqs. (3) and (4) describes inviscid fluid motions.

The dynamics of IGWs in the presence of wind $U(z)$ will be studied in the $(x, z)$ plane (i.e., in two-dimensional geometry). The flow velocity can be represented in the form $\boldsymbol{u}=(u+U(z), 0, w)$, where $u=-\partial \psi / \partial z, w=\partial \psi / \partial x$ and $\psi(t, x, z)$ is the stream function. The pressure $p$, density $\rho$ and potential temperature $\Theta$ are represented in the following form: $p=p_{0}(z)+\tilde{p}(t, x, z), \rho=\rho_{0}(z)+\tilde{\rho}(t, x, z)$, $\Theta=\Theta_{0}(z)+\tilde{\Theta}(t, x, z)$, where $p_{0}(z), \rho_{0}(z)$ and $\Theta_{0}(z)$ are the equilibrium pressure, density and potential temperature, while $\tilde{p}(t, x, z), \tilde{\rho}(t, x, z)$ and $\tilde{\Theta}(t, x, z)$ are corresponding perturbations.

From the momentum conservation in Eq. (3), we arrive at the following equation:

$$
\begin{aligned}
& \frac{\mathrm{d}}{\mathrm{d} \tau}\left(\nabla^{2} \psi+\frac{\mathrm{d} \ln \rho_{0}}{\mathrm{~d} z} \frac{\partial \psi}{\partial z}\right)-\left(U^{\prime \prime}+\frac{\mathrm{d} \ln \rho_{0}}{\mathrm{~d} z} U^{\prime}\right) \frac{\partial \psi}{\partial x} \\
& +J\left(\psi, \nabla^{2} \psi\right)=-\frac{\partial \chi}{\partial x}+\frac{1}{\rho_{0}^{2}} J(\tilde{\rho}, \tilde{p}),
\end{aligned}
$$

where $\mathrm{d} / \mathrm{d} \tau=\partial / \partial t+U \cdot \partial / \partial x, \quad \nabla^{2}=\partial^{2} / \partial x^{2}+\partial^{2} / \partial z^{2}$, $U^{\prime}=\mathrm{d} U / \mathrm{d} z, \quad U^{\prime \prime}=d^{2} U / \mathrm{d} z^{2}, \quad J(a, b)=(\partial a / \partial x) \partial b / \partial z-$ $(\partial a / \partial z) \partial b / \partial x$ is the Jacobian and $\chi=g \tilde{\rho} / \rho_{0}$. From the transport equation for potential temperature in Eq. (4), one can obtain

$$
\frac{\mathrm{d} \chi}{\mathrm{d} \tau}-\omega_{g}^{2} \frac{\partial \psi}{\partial x}+J(\psi, \chi)=0
$$

In the hydrostatic approximation, the equilibrium pressure decreases exponentially with height: $p_{0}\left(z-z_{0}\right)=$ $p_{0}\left(z_{0}\right) \exp \left[-\left(z-z_{0}\right) / H\right]$, where $p_{0}\left(z_{0}\right)$ is the pressure at a reference level $z_{0}, H=c_{\mathrm{s}}^{2} / \gamma g$ is the reduced height of the atmosphere and $c_{\mathrm{S}}=\left(\gamma p_{0} / \rho_{0}\right)^{1 / 2}$ is the sound speed. Let us assume that the equilibrium temperature $T_{0}$ and density $\rho_{0}$ depend on altitude as $T_{0}\left(z-z_{0}\right)=T_{0}\left(z_{0}\right) \exp \left[-\left(z-z_{0}\right) / L_{T}\right]$ and $\rho_{0}\left(z-z_{0}\right)=\rho_{0}\left(z_{0}\right) \exp \left[-\left(z-z_{0}\right) / L_{\rho}\right]$, where $L_{T}$ and $L_{\rho}$ are the temperature and density scales. The relation between $L_{T}$ and $L_{\rho}$ (i.e., $1 / L_{\rho}=1 / H-1 / L_{T}$ ) can be easily obtained from the equation of state for an ideal gas, $p(\rho T)^{-1}=$ const. Next, let us find the solutions of Eqs. (5) and (6) in the following form:

$\psi=\hat{\psi}(t, x, z) \exp \left(\frac{z-z_{0}}{2 L_{\rho}}\right)$,

and

$\chi=\hat{\chi}(t, x, z) \exp \left(\frac{z-z_{0}}{2 L_{\rho}}\right)$.

After substituting the Eqs. (7) and (8) into Eqs. (5) and (6), the equations for $\hat{\psi}(t, x, z)$ and $\hat{\chi}(t, x, z)$ become

$$
\begin{aligned}
& \frac{\mathrm{d}}{\mathrm{d} \tau}\left(\nabla^{2} \hat{\psi}-\frac{1}{4 L_{\varrho}^{2}} \hat{\psi}\right)-\left(U^{\prime \prime}-\frac{U^{\prime}}{L_{\rho}}\right) \frac{\partial \hat{\psi}}{\partial x} \\
& +J\left(\hat{\psi}, \nabla^{2} \hat{\psi}\right)=-\frac{\partial \hat{\chi}}{\partial x},
\end{aligned}
$$

and

$\frac{\mathrm{d} \hat{\chi}}{\mathrm{d} \tau}-\omega_{g}^{2} \frac{\partial \hat{\psi}}{\partial x}+J(\hat{\psi}, \hat{\chi})=0$. 
Equations (9) and (10) constitute the full set of reduced equations that describe the nonlinear dynamics of IGWs with spatial scales smaller than $2 L_{\rho}$ in the atmosphere with finite wind shear. In the limit of negligible wind effects and temperature gradients, Eqs. (9) and (10) coincide with those derived previously by Kaladze and Tsamalashvili (1997) and Stenflo and Shukla (2009). This set of equations also conserves the wave energy integral, $\partial E / \partial t=0$ where

$E=\int \rho_{0}\left[(\nabla \psi)^{2}+\frac{\psi^{2}}{4 L_{\rho}^{2}}+\frac{\chi^{2}}{\omega_{g}^{2}}\right] \mathrm{d} x \mathrm{~d} z$.

The system of Eqs. (9) and (10) can be reduced to the following equation:

$$
\begin{aligned}
& \frac{d^{2}}{\mathrm{~d} \tau^{2}}\left(\nabla^{2} \widehat{\psi}-\frac{1}{4 L_{\varrho}^{2}} \widehat{\psi}\right)-\left(U^{\prime \prime}-\frac{U^{\prime}}{L_{\varrho}}\right) \frac{d}{d \tau} \frac{\partial \widehat{\psi}}{\partial x}+\omega_{g}^{2} \frac{\partial^{2} \widehat{\psi}}{\partial x^{2}} \\
& +\frac{\mathrm{d}}{\mathrm{d} \tau} J\left(\widehat{\psi}, \nabla^{2} \widehat{\psi}\right)-\frac{\partial}{\partial x} J(\widehat{\psi}, \widehat{\chi})=0 .
\end{aligned}
$$

For perturbations in the form $\widehat{\psi}=\Psi(z) \exp \left(-i \omega t+i k_{x} x\right)$ and for $\left|U^{\prime \prime}\right| \gg U^{\prime} / L_{\varrho}$ Eq. (12) becomes the TaylorGoldstein (TG) equation (e.g., Dunkerton, 1997):

$$
\Psi^{\prime \prime}+\left(\frac{U^{\prime \prime}}{U-c}+\frac{\omega_{g}^{2}}{(U-c)^{2}}-k_{x}^{2}\right) \Psi=0,
$$

where $\Psi^{\prime \prime}=d^{2} \Psi / \mathrm{d} z^{2}$ and $c=\omega / k_{x}$ is the horizontal phase wave velocity. The role of the vertical wind shear on IGW absorption, reflection, refraction and entrapment at model boundary conditions in the atmosphere layer, described by the TG equation, has been investigated previously by Dunkerton (1997) and Yuan and Fritts (1989). In the neutrally stratified atmosphere, when the square of the BruntVäisälä frequency $\omega_{g}^{2}=0$, the TG equation reduces to the Orr-Sommerfeld (or Rayleigh) equation (e.g., Onishchenko et al., 2008) describing Kelvin-Helmholtz instability. By assuming the atmospheric perturbations in the form $\widehat{\psi}=$ $\widehat{\psi}_{k} \exp \left(-i \omega t+i k_{x} x+i k_{z} z\right)$, the Fourier transform of Eq. (12) gives the dispersion relation

$$
\left(k^{2}+\frac{1}{4 L_{\rho}^{2}}\right) \hat{\omega}^{2}+k_{x}\left(U^{\prime \prime}-\frac{U^{\prime}}{L_{\rho}}\right) \hat{\omega}=k_{x}^{2} \omega_{g}^{2},
$$

where $\hat{\omega}=\omega-k_{x} U$ is the wave frequency in the reference frame moving with the wind and $k^{2}=k_{x}^{2}+k_{z}^{2}$, and $k_{(x, z)}$ are the $(x, z)$ components of the wave vector $\boldsymbol{k}$.

In the shortwave limit approximation $k^{2} L_{\rho}^{2} \gg 1$ and by assuming that $U^{\prime \prime}=U / L_{U}^{2}$, where $L_{U}$ is the characteristic spatial scale of the wind shear from Eq. (14), one can obtain the condition for instability of IGWs:

$$
\frac{H}{L_{T}}>\frac{\gamma-1}{\gamma}+\frac{\gamma}{4} \frac{U^{2}}{c_{\mathrm{s}}^{2}} \frac{H^{4}}{L_{U}^{4}} \frac{1}{k_{z}^{2} H^{2}} .
$$

Note that this condition has been obtained by presuming that $L_{U} \ll L_{\rho}$ (i.e., the characteristic spatial scale of the wind shear is much smaller than density scales). The inequality in Eq. (15) is the condition of stability of IGW in the Earth's atmosphere with the shear wind. From Eq. (15) it follows that in such an atmosphere the threshold of instability of IGW wave increases, due to the presence of the second term on the right-hand side.

\section{Convective cells}

In this section we derive the stationary solutions of the nonlinear Eqs. (9) and (10) in the reference frame moving, with the velocity $v$ along the $x$ direction. Let us introduce the new variable $\eta=x-v t$. By substituting $\eta$ into Eqs. (9) and (10), Eq. (9) becomes

$$
\begin{aligned}
& (U-v) \frac{\partial}{\partial \eta}\left(\nabla^{2} \hat{\psi}-\frac{1}{4 L_{\varrho}^{2}} \hat{\psi}\right)+\left(U^{\prime \prime}-\frac{U^{\prime}}{L_{\rho}}\right) \frac{\partial \hat{\psi}}{\partial \eta} \\
& +J\left(\hat{\psi}, \nabla^{2} \hat{\psi}\right)+\frac{\partial \hat{\chi}}{\partial \eta}=0 .
\end{aligned}
$$

Making use of the respective particular solution of Eq. (10),

$\hat{\chi}=\frac{\omega_{g}^{2}}{U-v} \hat{\psi}$,

from Eq. (16) we obtain

$$
\begin{aligned}
& (U-v)^{2} \frac{\partial}{\partial \eta}\left(\nabla^{2} \hat{\psi}-\frac{1}{4 L_{\varrho}^{2}} \hat{\psi}\right)+(U-v)\left(U^{\prime \prime}-\frac{U^{\prime}}{L_{\rho}}\right) \frac{\partial \hat{\psi}}{\partial \eta} \\
& +(U-v) J\left(\hat{\psi}, \nabla^{2} \hat{\psi}\right)+\omega_{g}^{2} \frac{\partial \hat{\psi}}{\partial \eta}=0 .
\end{aligned}
$$

By introducing the differential operator $J_{1}[a, b]=$ $(\partial a / \partial \eta) \partial b / \partial z-(\partial a / \partial z) \partial b / \partial \eta$, the Eq. (18) can be written in the following form:

$J_{1}\left[\nabla^{2} \hat{\psi}-\Lambda \hat{\psi}, \hat{\psi}+(U-v) z\right]=0$,

where

$\Lambda=\frac{1}{4 L_{\varrho}^{2}}-\frac{1}{U-v}\left(U^{\prime \prime}-\frac{U^{\prime}}{L_{\rho}}\right)-\frac{\omega_{g}^{2}}{(U-v)^{2}}$.

Here we consider that $U-v=$ const and $\Lambda=$ const.

In case, when wind velocity and wind shear are assumed to be zero (i.e., $U, U^{\prime}, U^{\prime \prime} \rightarrow 0$ ), Eqs. (19) and (20) reduce to those obtained by Kaladze and Tsamalashvili (1997), Stenflo and Shukla (2009), and Onishchenko et al. (2013). An equation similar to Eq. (19) has been studied analytically and numerically previously (e.g., Onishchenko et al., 2008). The authors of this paper have shown that this equation yields a solution in the form of quasi-periodical, in the horizontal 

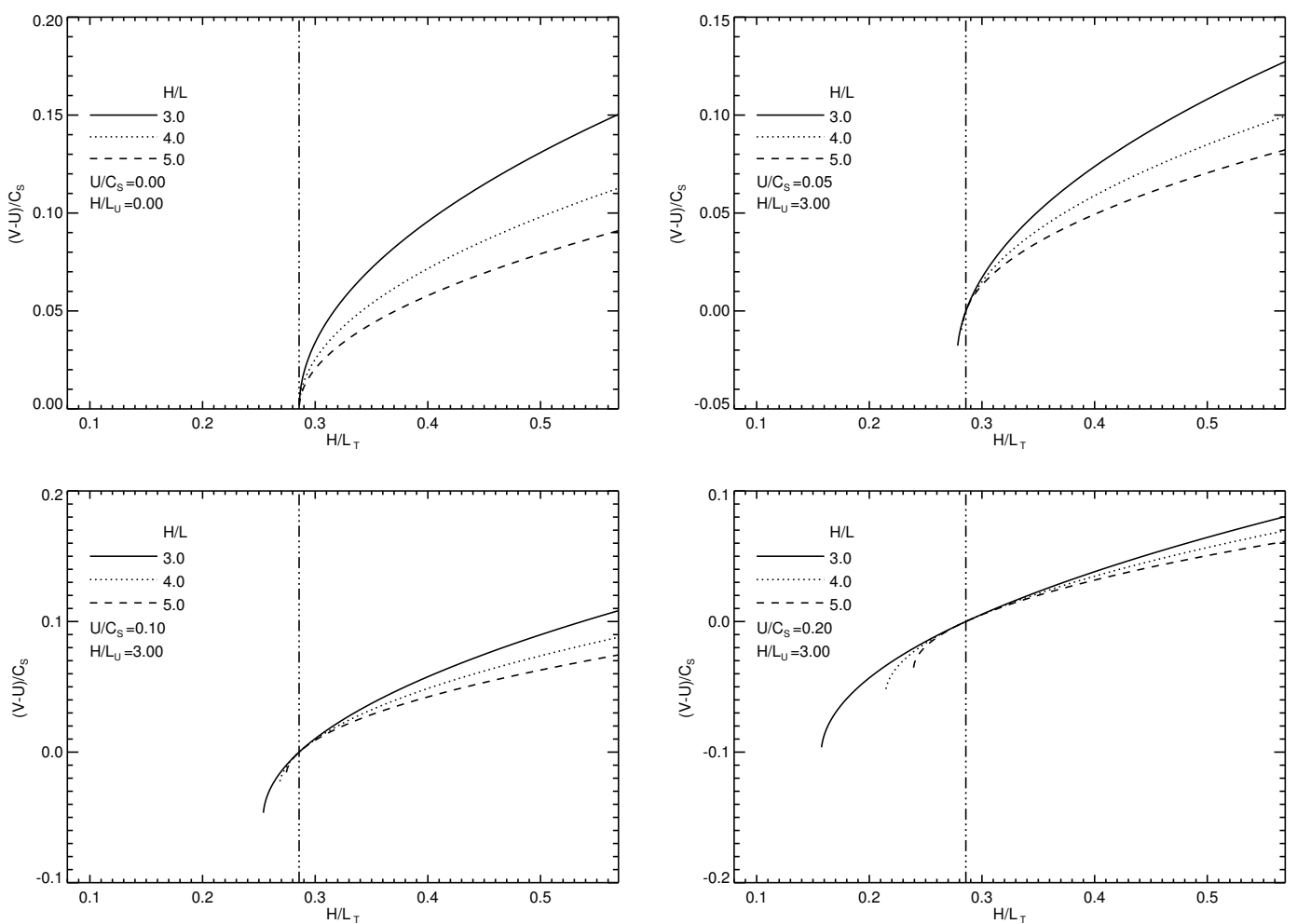

Fig. 1. The roll normalized velocity $(v-U) / c_{S}$ as a function of the dimensionless parameters $H / L, H / L_{T}$, normalized wind velocity $U / c_{\mathrm{S}}$ and the characteristic spatial scale of the wind shear $H / L_{U}$.

plain, vortex streets (the so-called "cat's eyes") and dipole vortices.

We are interested in the solution of Eq. (19) in the form of the vortex structures (convective cells) such as dipole vortices or vortex streets with incompressible flow, $\nabla \cdot v=0$, and finite vorticity, $(\nabla \times \boldsymbol{v})_{y}=-\nabla^{2} \psi+U^{\prime}$. Vortex structures, elongated along the axis $y$, have the form of rolls. In the outer region of the rolls, we have

$\nabla^{2} \hat{\psi}-\Lambda \hat{\psi}=0$

The solution of Eq. (21) is localized in space if $\Lambda>0$. For numerical estimates we approximate the second derivative in the form $U^{\prime \prime}=U^{\prime} / L_{U}$, where $L_{U} \ll L_{\rho}$. Next, from Eq. (21) one obtains the equation for the roll velocity $v$, i.e.,

$$
\left[1-\frac{1}{4}\left(\frac{L}{L_{\rho}}\right)^{2}\right](U-v)^{2}+\frac{L^{2} U^{\prime}}{L_{U}}(U-v)+L^{2} \omega_{g}^{2}=0
$$

Note that Eq. (22) includes the characteristic spatial scale of the roll $L=\Lambda^{-1 / 2}$ and atmosphere parameters $H, L_{T}$, the Brunt-Väisälä frequency $\omega_{g}$, wind velocity $U$ and wind shear $U^{\prime}$. From Eq. (22) it follows that, in the stably stratified atmosphere without sheared wind, the characteristic scale of the rolls should be $L<2 L_{\rho}$. In the shallow atmosphere with sheared wind, the vortex structures exist if

$R i<\frac{1}{4} \frac{L^{2}}{L_{U}^{2}}\left(1-\frac{1}{4} \frac{L^{2}}{L_{\rho}^{2}}\right)^{-1}$

is satisfied, and therefore the roll structures can originate only in a dynamically unstable atmosphere. The inequality (Eq. 23) can be represented as

$\frac{H}{L_{T}}>\frac{\gamma-1}{\gamma}-\frac{\gamma}{4} \frac{U^{2}}{c_{\mathrm{s}}^{2}} \frac{H^{4}}{L_{U}^{4}} \frac{L^{2}}{H^{2}}$

Comparison of Eqs. (15) and (24) shows that these inequalities coincide with the substitution $k_{z}^{2}=-1 / L^{2}$. One can see from the condition in Eq. (24) that, by neglecting the shear wind (i.e., if $U=0$ and $L_{U} \rightarrow \infty$ ) in the convectively unstable atmosphere, the roll structure may exist if $\omega_{g}^{2}<0$ for super-adiabatic temperature profile, i.e., $H / L_{T}>$ $(\gamma-1) / \gamma$ (see Onishchenko et al., 2013).

The estimations of characteristic vertical temperature and wind profiles in the nocturnal mesopause region $(80-105 \mathrm{~km})$ have been obtained during long-term lidar observations (e.g., Sherman and Che, 2006; Zhao, 2003). The authors have found that temperature profile possesses near-adiabatic lapse rate, $H / L_{T}>(\gamma-1) / \gamma$, in this region. The mesosphere at altitudes $80-82 \mathrm{~km}$ was convectively unstable, $R i<0$. In the layer at altitudes $90-93 \mathrm{~km}$, maximum winds and wind 
shears were observed for all seasons except summer. The winter wind shears were larger than those observed in other seasons; $35 \%$ of the winter profiles at this altitude contained wind shears exceeding $40 \mathrm{~m} \mathrm{~s}^{-1}$ per kilometer. Such large shears, when coupled with the sufficient negative temperature gradient at this height, often push the local atmosphere into dynamic instability, $R i<1 / 4$.

The solutions of Eq. (18), for two different cases - with and without presence of the wind shear - are plotted in Fig. 1. The normalized minimal roll velocity $v / c_{\mathrm{S}}$ as a function of $H / L_{T}$ in the atmosphere without wind shear for $H / L=3$, 4 and 5 is shown in Fig. 1a. For calculation, the ratio of specific heats has been chosen to be $\gamma=1.4$. Figure $1 \mathrm{~b}, \mathrm{c}$ and $d$ show that the dependences of the minimum absolute value of the relative velocity of rolls for the values of the atmospheric parameters are close to those observed by Sherman and Che (2006) and Zhao (2003), and the wind shear can significantly change the condition of the existence of the roll structures. Also, from inequality (Eq. 24), it follows that the roll structures can exist in the convectively stable atmosphere with a sub-adiabatic temperature profile. The vertical dashed triple-dotted line (see Fig. 1.) corresponds to the equality, $H / L_{T}=(\gamma-1) / \gamma$, and separates the convectively stable atmosphere region with positive Richardson numbers (left side) from the convectively unstable atmosphere with negative Richardson numbers (right of the line). From this figure it is seen that, in the convectively stable atmosphere with sheared winds, the roll velocity in the wind reference frame is negative while in the convectively unstable atmosphere it is positive (the direction of roll speed coincides with the direction of wind). In the convectively unstable atmosphere, the roll velocity weakly depends on the spatial scale of the rolls and on the parameter $H / L_{T}$.

\section{Conclusions}

The present theory generalizes the recent study of Onishchenko et al. (2013), in which the roll-type vortex structures have been studied without taking into account the shear winds in the atmosphere with vertical temperature gradients. Onishchenko et al. (2013) have shown that vortices of IGWs with spatial scale $L$ smaller than the reduced height $H$ can originate only in a convectively unstable atmosphere. In this paper we have demonstrated that the wind shear is important for the formation of the roll structure. Also, we have shown that the obtained set of nonlinear equations in the quasistationary case can be reduced to the equation that yields a vortex solution. The relation that connects roll parameters (spatial scale $L$ and horizontal speed $v$ ) with atmosphere and shear wind parameters has been derived. We have found that roll-type vortex structures of the IGWs can exist in the dynamically unstable atmosphere.
Acknowledgements. This research is partially supported by the International Space Science Institute in Bern (Switzerland) through the grant "Large-scale vortices and zonal winds in planetary atmospheres/ionospheres: Theory vs observations", RFBR through the grant 14-05-00850, and STFC. V. Fedun is grateful to The Royal Society (International Exchanges Scheme). W. Horton is supported by NSF grant 0964692 to the University of Texas at Austin, the University of Aix-Marseille/CNRS, and by the IMeRA Grant for Advanced Research.

Topical Editor K. Hosokawa thanks B. Eliasson and one anonymous referee for their help in evaluating this paper.

\section{References}

Dunkerton, T. J.: (1997), Shear instability of internal inertiagravity waves, J. Atmos. Sci., 54, 1628-1641, doi:10.1175/15200469(1997)054<1628:SIOIIG>2.0.CO;2, 1997.

Dutton, J. A.: Dynamics of atmospheric motions, Dover Publications, NY, 640 pp., 1986.

Fritts, D. C., and Alexander, M.: Gravity wave dynamics and effects in the middle atmosphere, Rev. Geophys., 41, 3-1, doi:10.1029/2001RG000106, 2003.

Horton, W., Kaladze, T. D., van Dam, J. W., and Garner, T. W.: Zonal flow generation by internal gravity waves in the atmosphere, J. Geophys. Res., 113, A08312, doi:10.1029/2007JA012952, 2008.

Kaladze, T. D. and Tsamalashvili, L. V.: Solitary dipole vortices in the Earth's ionosphere, Phys. Lett. A, 232, 269-274, doi:10.1016/S0375-9601(97)00377-0, 1997.

Lu, X., Liu, A. Z., Swenson, G. R., Li, T., Leblanc, T. and McDermid, I. S.: Gravity wave propagation and dissipation from the stratosphere to the lower thermosphere, J. Geophys. Res., 114, D11101, doi:10.1029/2008JD010112, 2009.

Mitchell, N. J., and Howells, V. S. C.: Vertical velocities associated with gravity waves measured in the mesosphere and lower thermosphere with the EISCAT VLF radar, Ann. Geophys., 16, 1367-1379, doi:10.1007/s00585-998-1367-0, 1998.

Onishchenko, O. G. and Pokhotelov, O. A.: Generation of zonal structures by internal gravity waves in the Earth's atmosphere, Doklady Earth Sciences, 445, 845-848, doi:10.1134/S1028334X12070070, 2012.

Onishchenko, O., Pokhotelov, O., and Astafieva, N.: Generation of large-scale eddies and zonal winds in planetary atmospheres, Physics-Uspekhi, 51, 577-589, doi:10.1070/PU2008v051n06ABEH006588, 2008.

Onishchenko, O., Pokhotelov, O., and Fedun, V.: Convective cells of internal gravity waves in the earth's atmosphere with finite temperature gradient, Ann. Geophys., 31, 459-462, doi:10.5194/angeo-31-459-2013, 2013.

Picard, R. H., O’Neil, R. R., Gardiner, H. A., Gibson, J., Winick, J. R., Gallery, W. O., Stair Jr., A. T., Wintersteiner, P. P., Hegblom, E. R., and Richards, E.: Remote sensing of discrete stratospheric gravity-wave structure at $4.3-\mu \mathrm{m}$ from the MSX satellite, Geophys. Res. Lett., 25, 2809-2812, doi:10.1029/98GL01701, 1998.

Sherman, J. P. and Che, C. Y.: Seasonal variation of mesopause region wind shears, convective and dynamic instabilities above Fort Collins, CO: A statistical study, J. Atmos. Solar-Terr. Phys., 68, 1061-1074, doi:10.1016/j.jastp.2006.01.011, 2006. 
Šindelářová, T., Burešová, D., and Chum, J.: Observations of acoustic-gravity waves in the ionosphere generated by severe tropospheric weather, Stud. Geophys. Geod., 53, 403-418, doi:10.1007/s11200-009-0028-4, 2009.

Stenflo, L. and Shukla, P. K.: Nonlinear acoustic-gravity waves, J. Plasma Phys., 75, 841-847, doi:10.1017/S0022377809007892, 2009.

Swenson, G. R. and Espy, P. J.: Observations of 2-dimensional airglow structure and $\mathrm{Na}$ density from the ALOHA, October 9, 1993 "storm flight", Geophys. Res. Lett., 22, 2845-2848, doi:10.1029/95GL02795, 1995.
Yuan, L. and Fritts, D., C.: Influence of a mean shear on the dynamical instability of an inertio-gravity wave, J. Atmosp. Sci., 46, 2562-2568, doi:10.1175/15200469(1989)046<2562:IOAMSO>2.0.CO;2, 1989.

Zhang, F.: Generation of mesoscale gravity waves in uppertropospheric jet-front systems, J. Atmos. Sci., 61, 440-457, doi:10.1175/1520-0469(2004)061<0440:GOMGWI>2.0.CO;2, 2004.

Zhao, Y., Liu, A. Z., and Gardner, C. S.: Measurements of atmospheric stability in the mesopause region at starfire optical range, NM, J. Atmosp. Solar Terr. Phys., 65, 219-232, doi:10.1016/S1364-6826(02)00288-2, 2003 